Supporting information for

\title{
Probing the interior of peptide amphiphile supramolecular aggregates
}

\author{
John D. Tovar, ${ }^{1,2}$ Randal C. Claussen ${ }^{3}$ and Samuel I. Stupp ${ }^{* 1,2,3,4}$
}

\begin{abstract}
${ }^{1}$ Department of Materials Science and Engineering; ${ }^{2}$ Institute for BioNanotechnology in Medicine (IBNAM); ${ }^{3}$ Department of Chemistry; ${ }^{4}$ Feinberg School of Medicine; Northwestern University, Evanston, IL 60208.
\end{abstract}

\section{Contents:}

Circular dichroism spectra of PAs 1 and 2 (Figures S1-S2)

Circular dichroism of PAs 3,4 and 5 diluted with a 40-fold excess of PA 6 (Figures S3-S5)

Stern-Volmer plots of coassemblies of PAs 4 and 5 diluted with PA 6 (Figures S6-S7)

NMR spectra of PAs (Figures S8-S12)

UV-vis spectra of PAs 1 and 2 (Figures S13-S14)

UV-vis and fluorescence of 1:40 coassemblies of PAs 3,4 and 5 with PA 6 (Figures S15-S17)

UV-vis and fluorescence of pyrene acetic acid and 40-fold excess of PA 6 (Figure S18)

Transmission electron micrographs of PA aggregates (Figures S19-S23) 


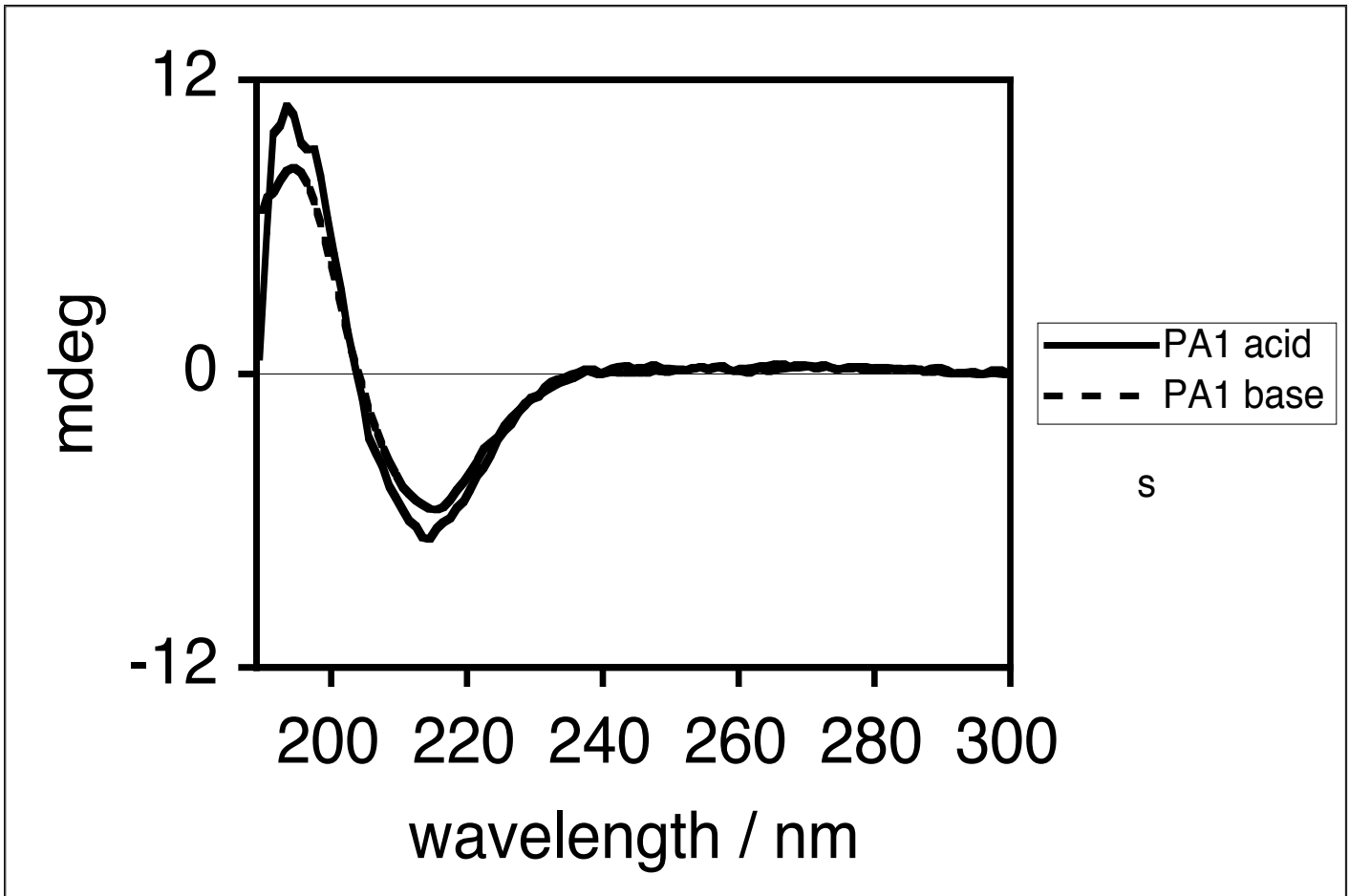

Figure S1: Circular dichroism (CD) of PA 1 in water under basic and acidic conditions.

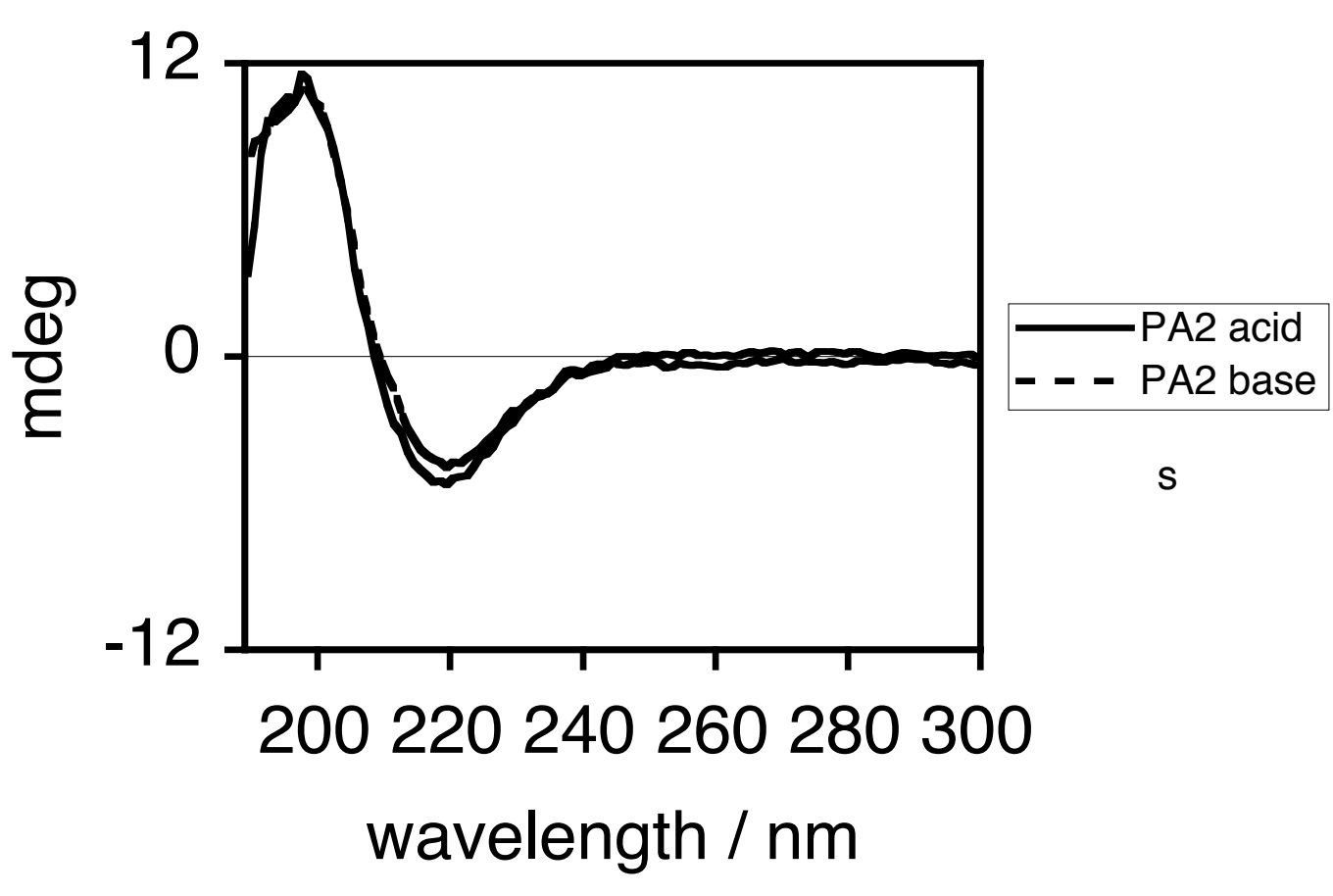

Figure S2: Circular dichroism (CD) of PA 2 in water under basic and acidic conditions. 


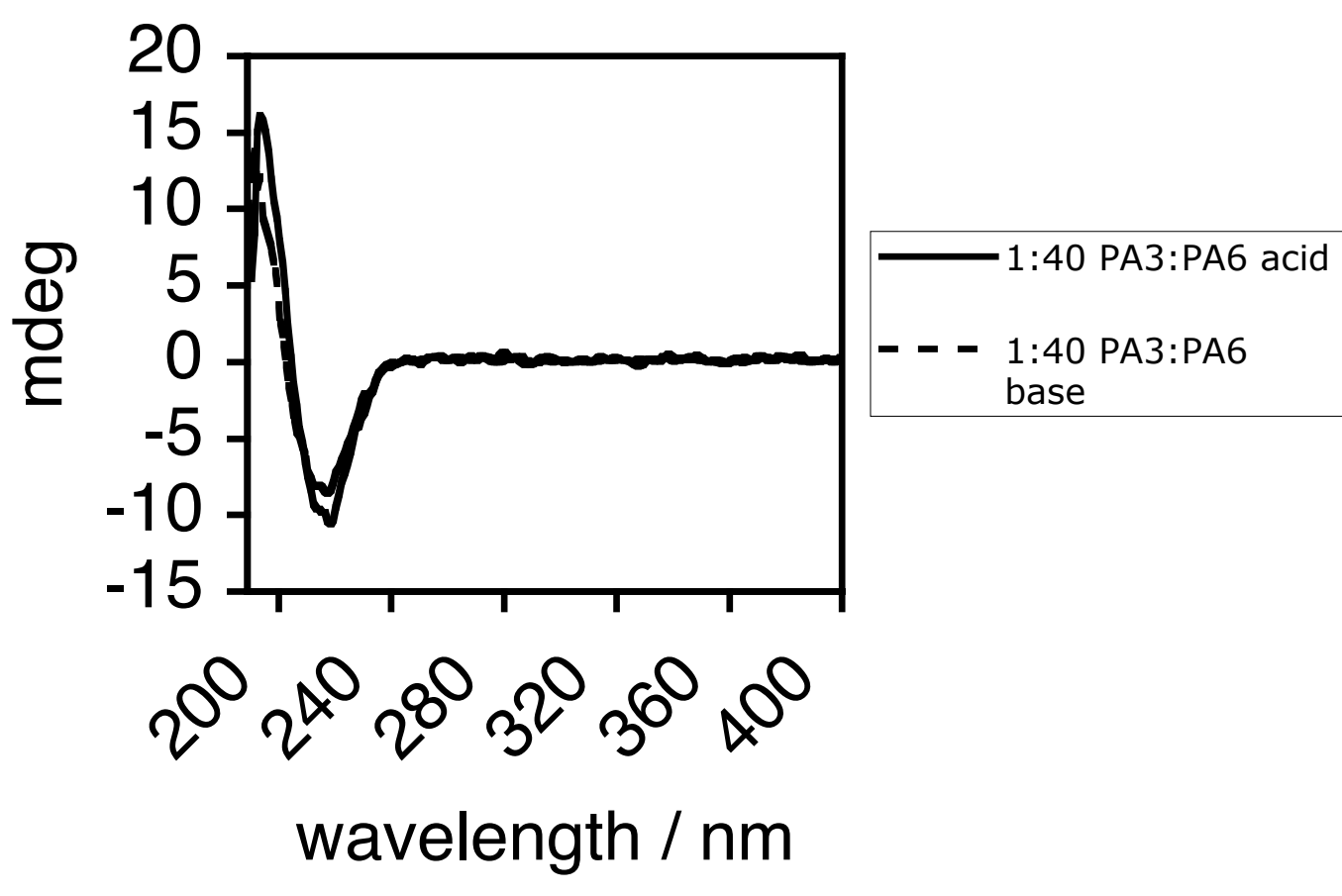

Figure S3: CD of a 1:40 solution of PA 3 and PA 6 in water under acidic and basic conditions

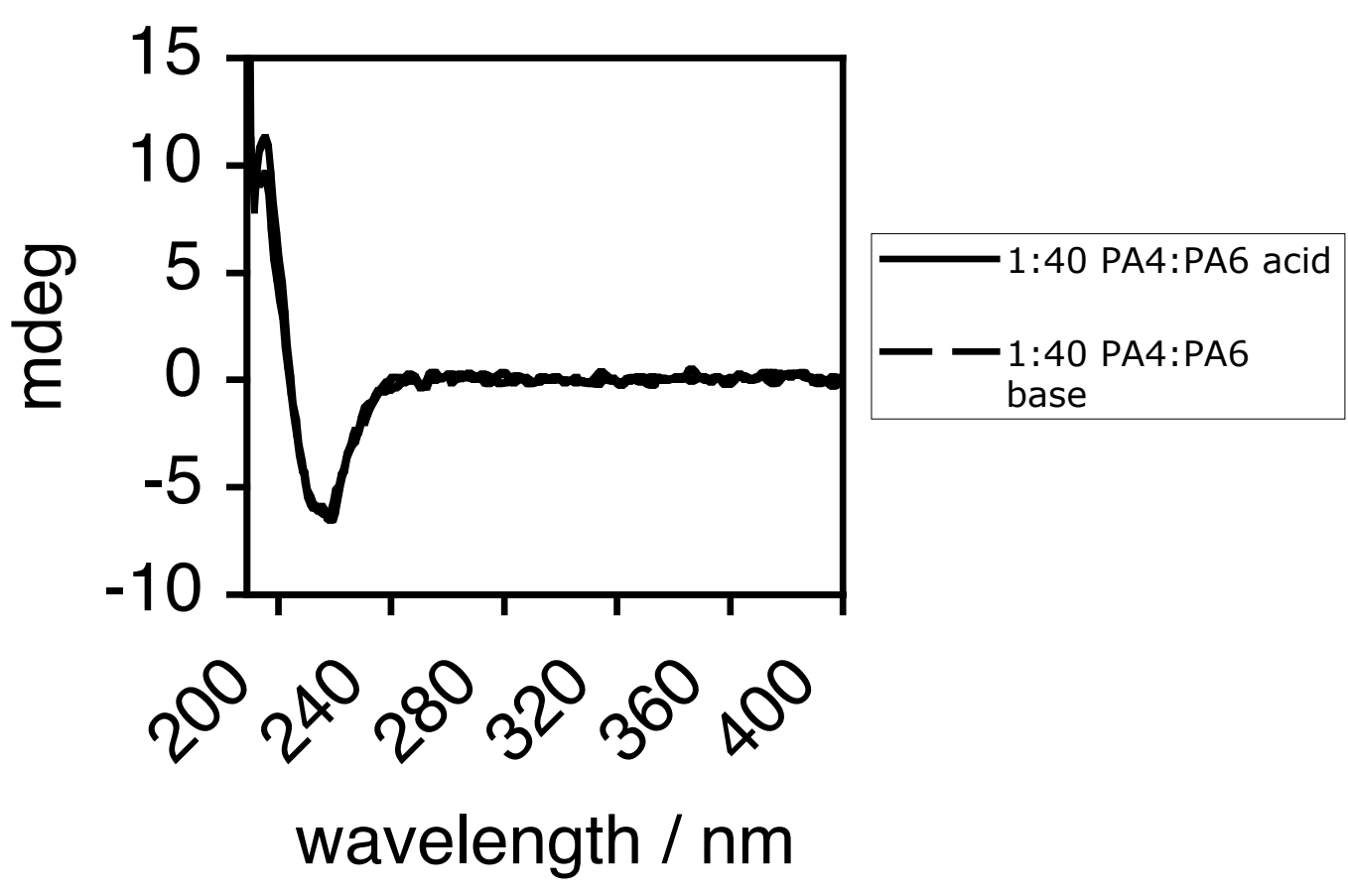

Figure S4: CD of a 1:40 solution of PA 4 and PA 6 in water under acidic and basic conditions 


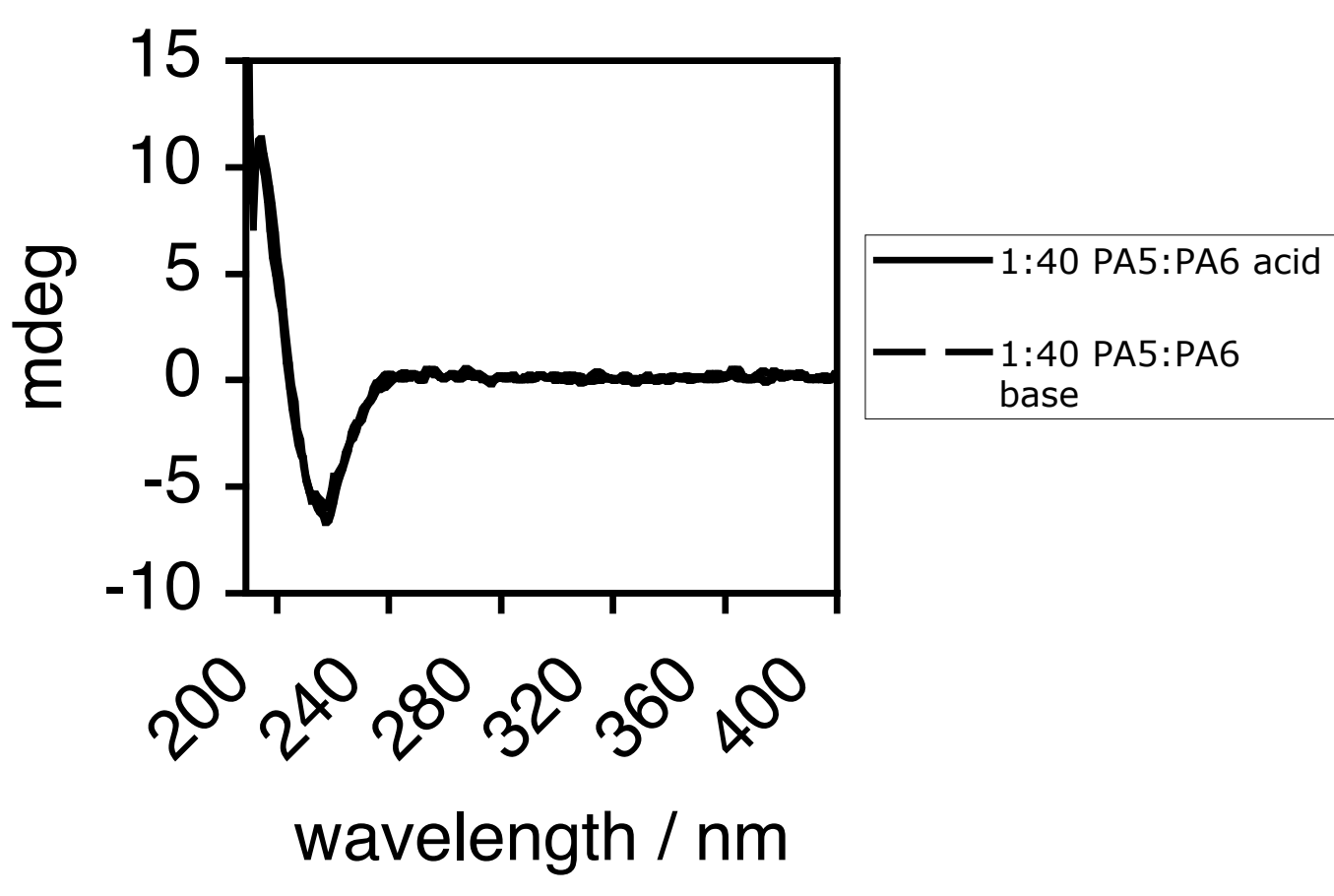

Figure S5: CD of a 1:40 solution of PA 5 and PA 6 in water under acidic and basic conditions 


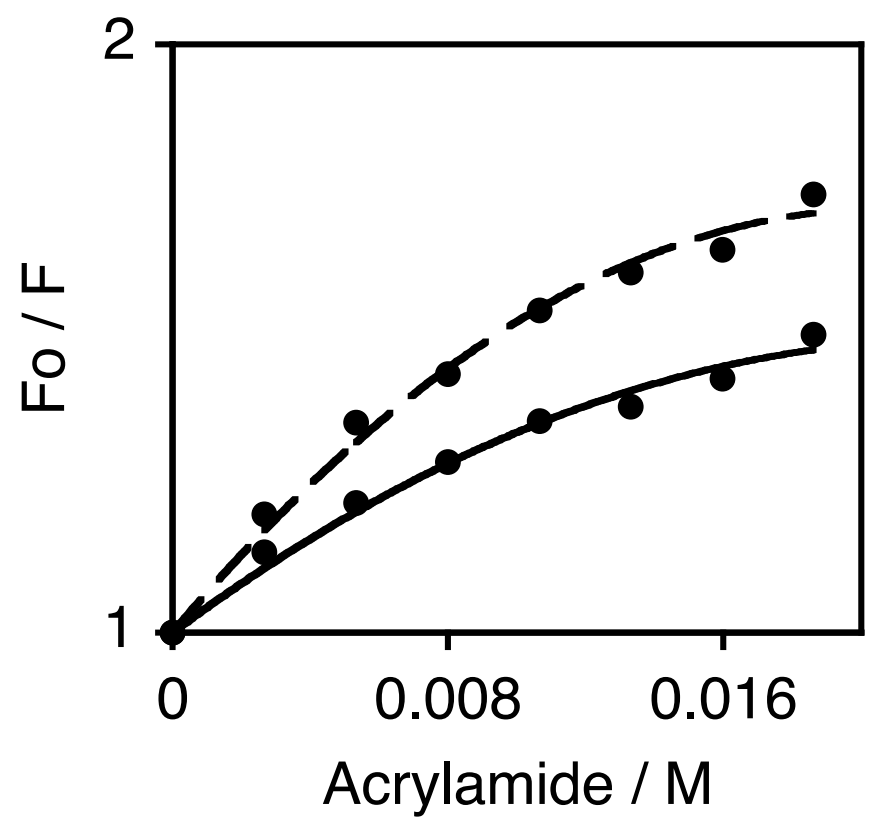

Figure S6: Stern-Volmer quenching response for 1:40 ratio of PA 4 : PA 6 under acidic (-) and basic (- - -) conditions.

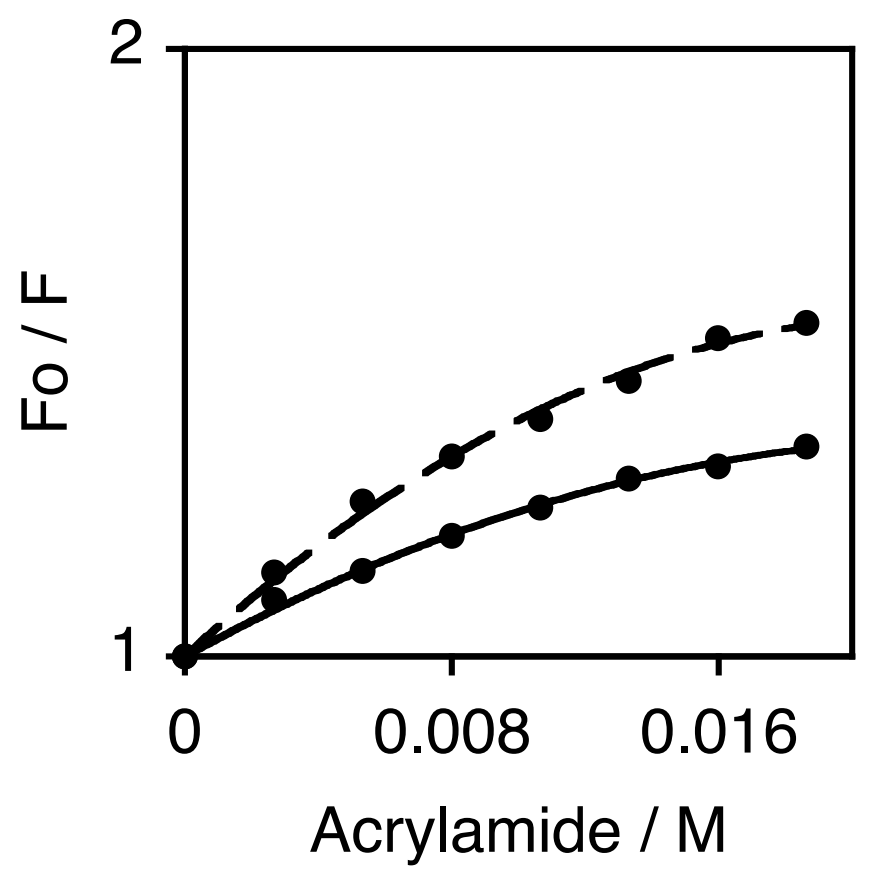

Figure S7: Stern-Volmer quenching response for 1:40 ratio of PA 5 : PA 6 under acidic (-) and basic (- - -) conditions. 
Figure S8: ${ }^{1} \mathrm{H}$ NMR spectrum of PA $1\left(400 \mathrm{MHz}, \mathrm{D}_{2} \mathrm{O}\right)$. 
Figure S9: ${ }^{1} \mathrm{H}$ NMR spectrum of PA $2\left(400 \mathrm{MHz}, d_{6}\right.$-DMSO) 
Figure S10: ${ }^{1} \mathrm{H}$ NMR spectrum of PA 3 (400 MHz, $d_{6}$-DMSO) 
Figure S11: ${ }^{1} \mathrm{H}$ NMR spectrum of PA $3\left(400 \mathrm{MHz}, d_{6}\right.$-DMSO) 
Figure S12: ${ }^{1} \mathrm{H}$ NMR spectrum of PA 5 (400 MHz, $d_{6}$-DMSO). 


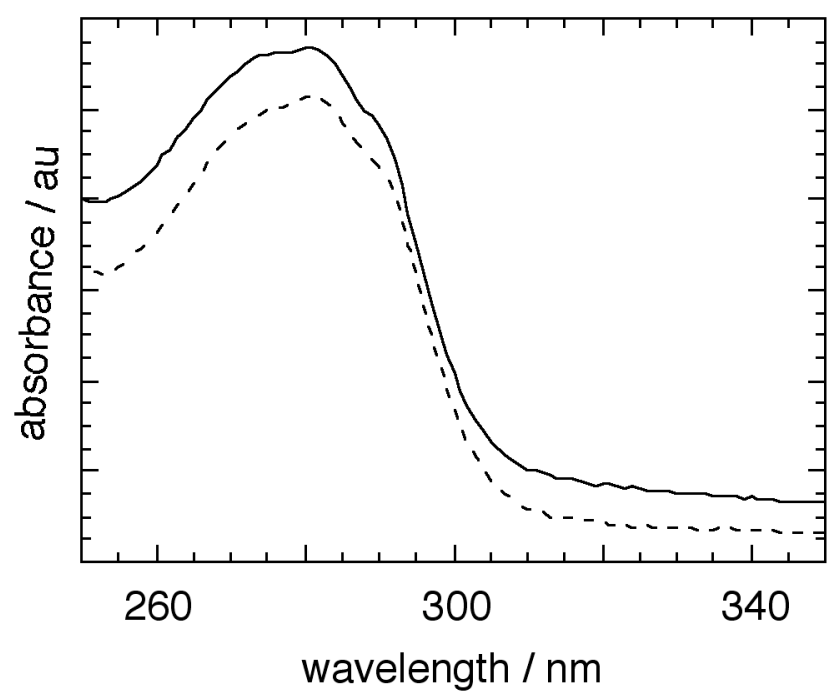

Figure S13: UV-vis of PA 1 under basic ( - - -) and acidic ( - ) conditions.

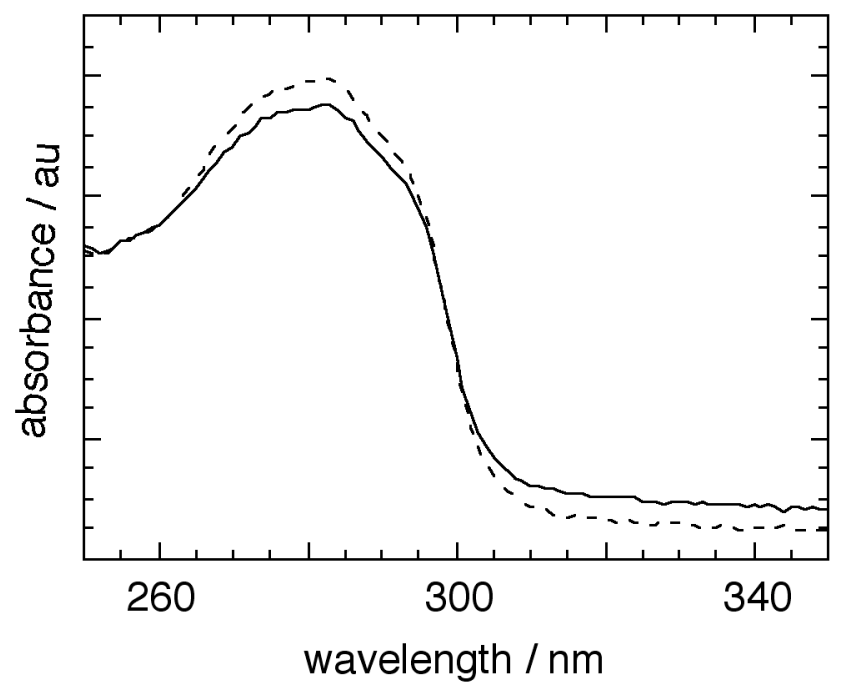

Figure S14: UV-vis of PA 2 under basic ( - - -) and acidic (-) conditions. 


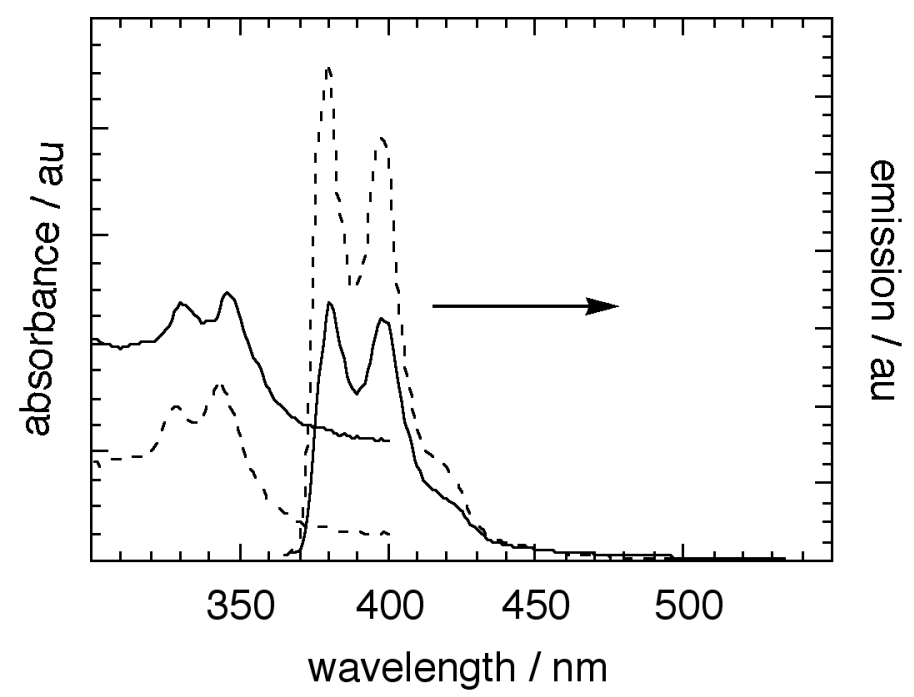

Figure S15: UV-vis and fluorescence of 1:40 solutions of PA 3 and PA 6 under basic ( - - - ) and acidic $(-)$ conditions.

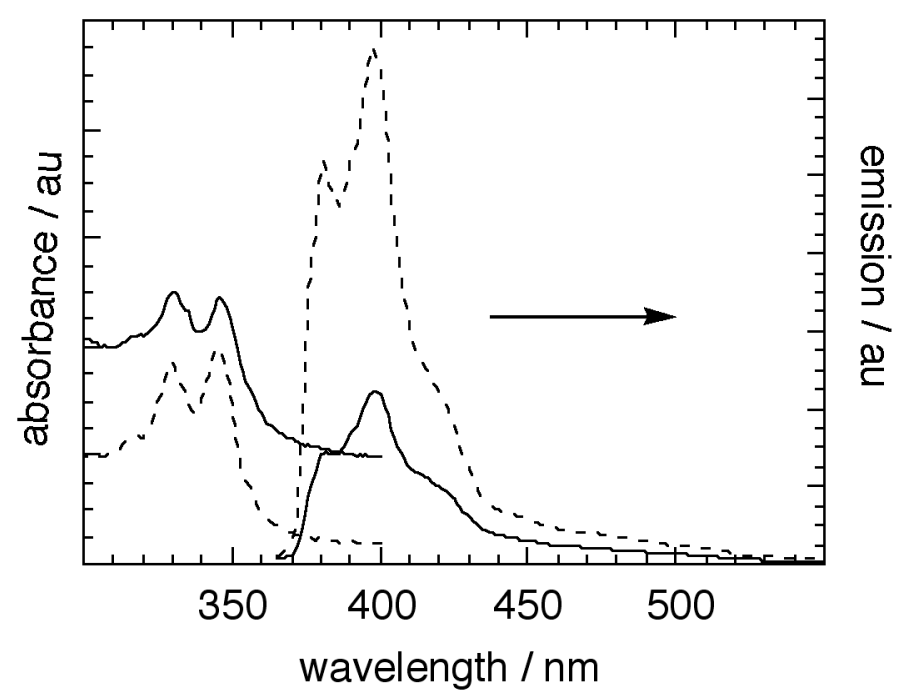

Figure S16: UV-vis and fluorescence of 1:40 solutions of PA 4 and PA 6 under basic ( - - - ) and acidic $(-)$ conditions. 


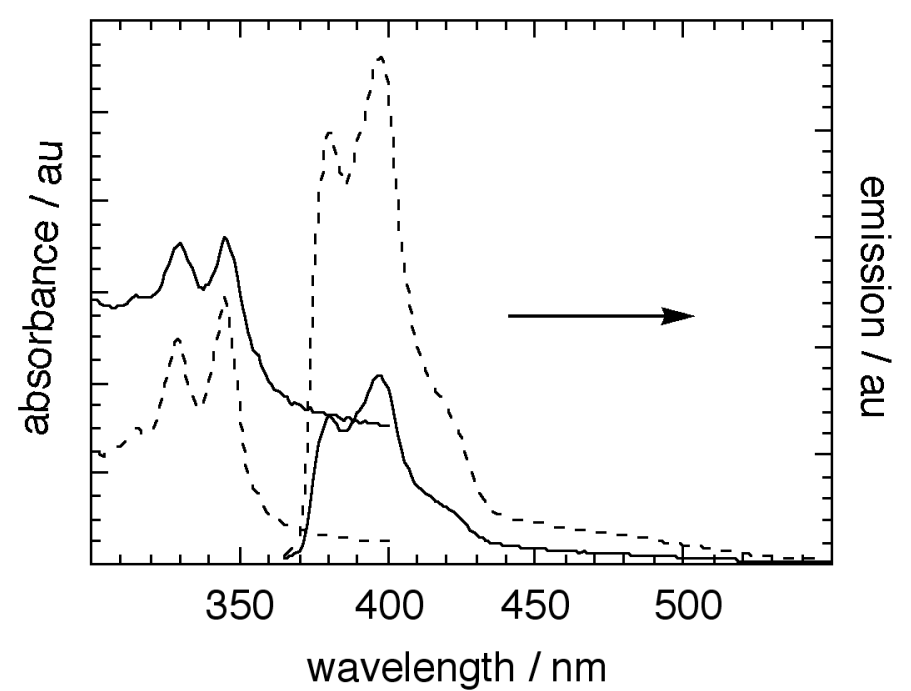

Figure S17: UV-vis and fluorescence of 1:40 solutions of PA 5 and PA 6 under basic ( - - - -) and acidic ( $(-)$ conditions.

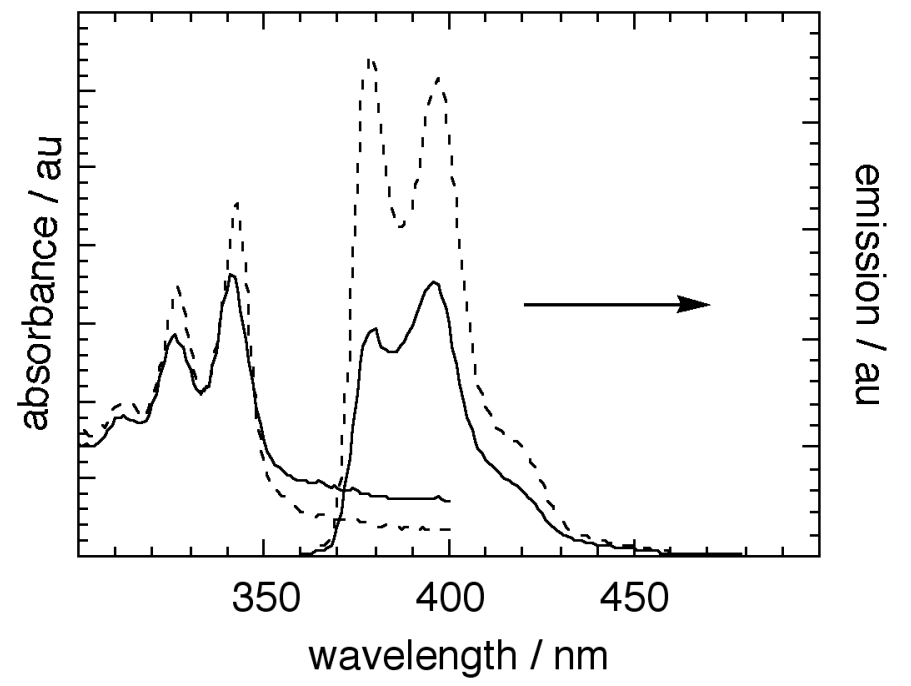

Figure S18: UV-vis and fluorescence of 1:40 solutions of pyrene acetic acid and PA 6 under basic ( - - -) and acidic ( - ) conditions. 


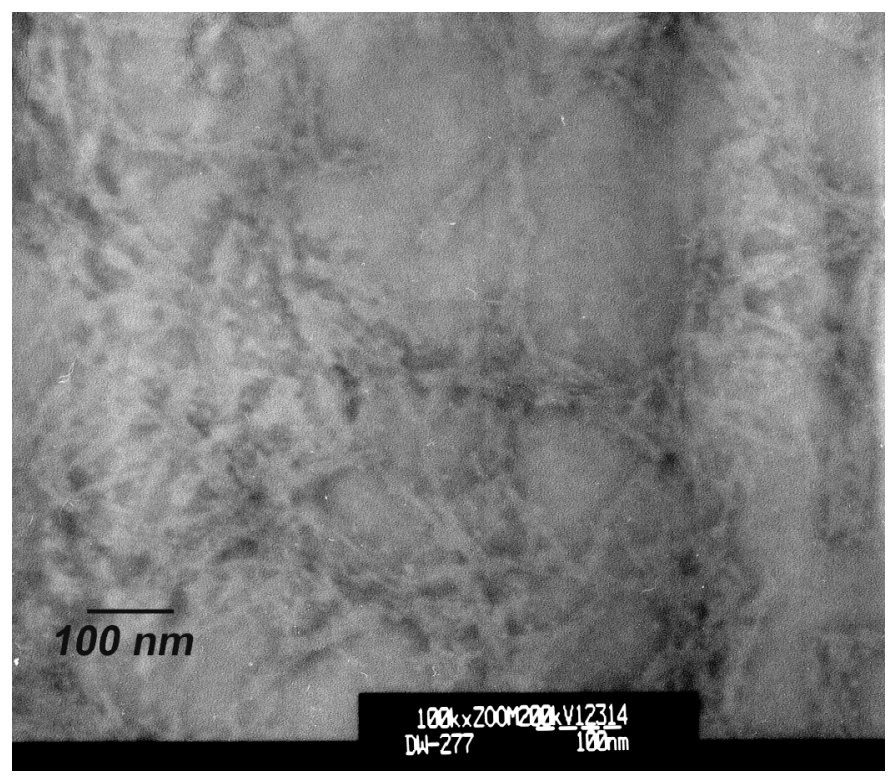

Figure S19: Transmission electron microscopy of assembled aggregates made up of PA 1, negatively stained with phosphotungstic acid (100,000x magnification).

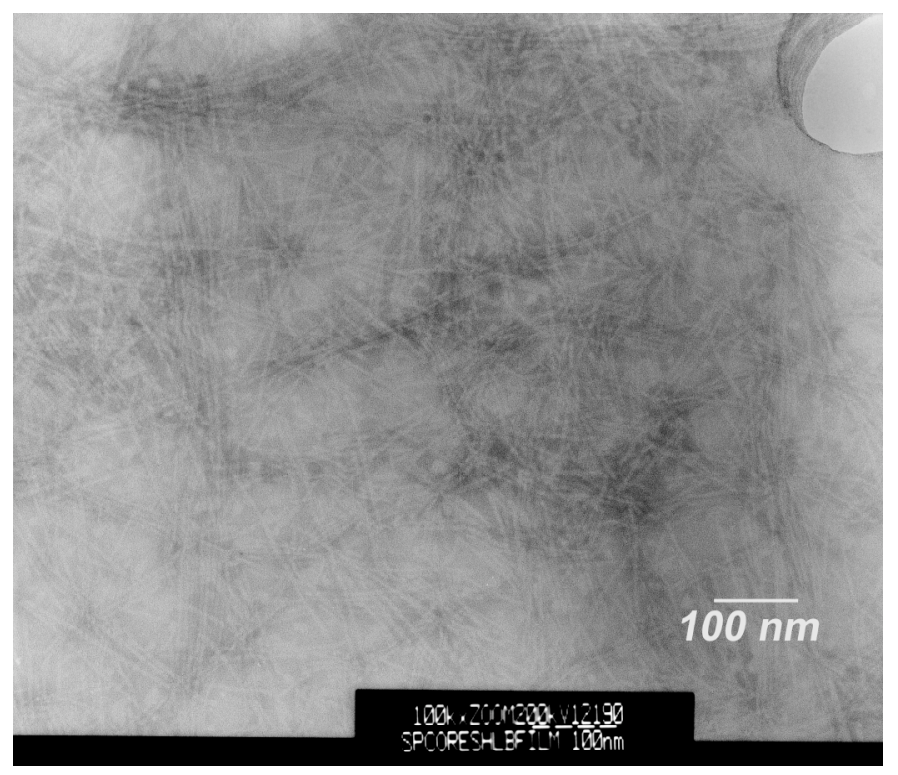

Figure S20: Transmission electron microscopy of assembled aggregates made up of PA 2, negatively stained with phosphotungstic acid (100,000x magnification). 


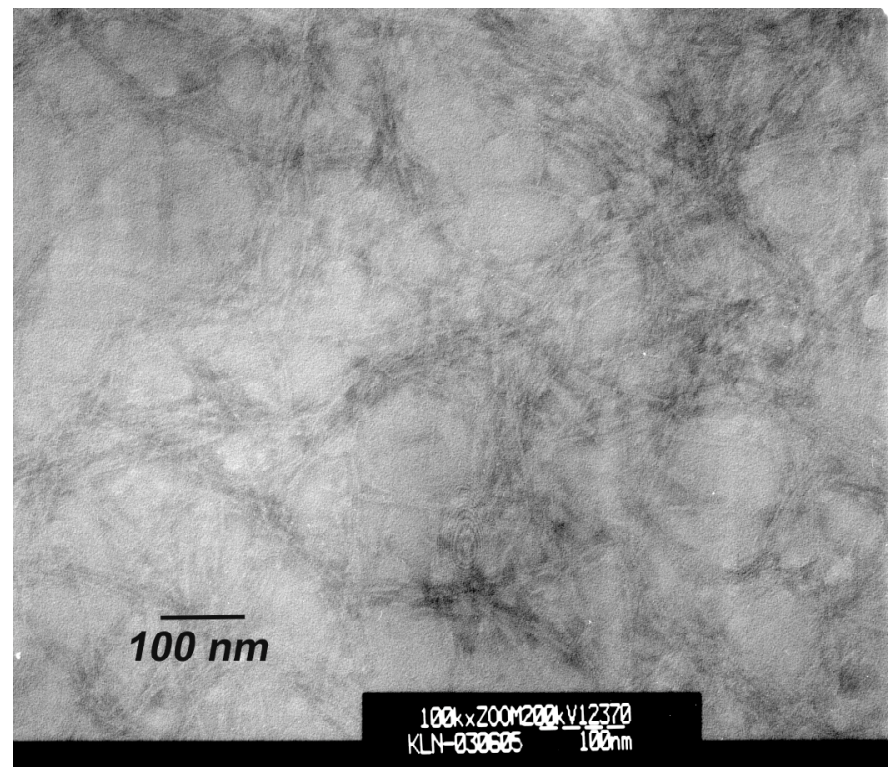

Figure S21: Transmission electron micrograph of assembled aggregates made up of a 40-fold excess of PA 6 mixed with PA 3, negatively stained with phosphotungstic acid (100,000x magnification).

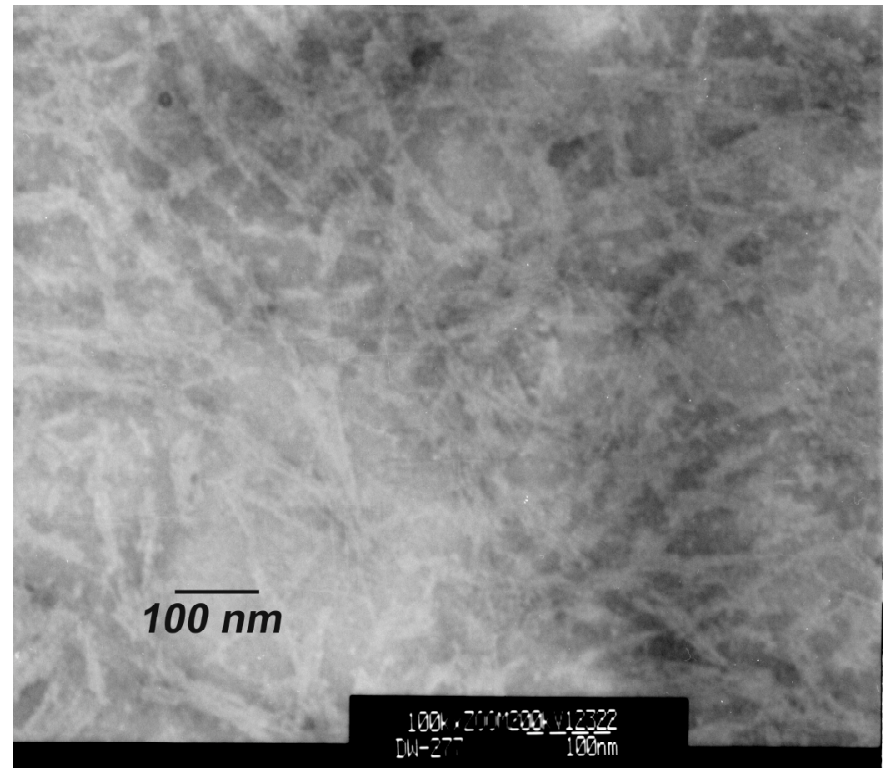

Figure S22: Transmission electron micrograph of assembled aggregates made up of a 40-fold excess of PA 6 mixed with PA 4, negatively stained with phosphotungstic acid (100,000x magnification). 


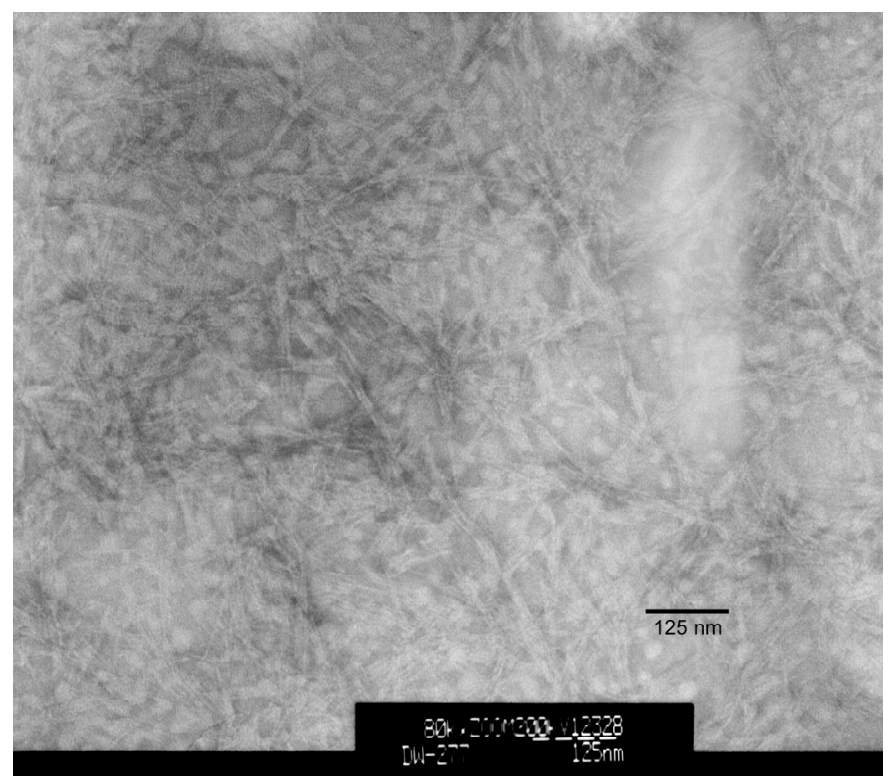

Figure S23: Transmission electron micrograph of assembled aggregates made up of a 40-fold excess of PA 6 mixed with PA 5, negatively stained with phosphotungstic acid (80,000x magnification). 